\title{
Jogo de Física de partículas: Descobrindo o bóson de Higgs
}

\author{
Particle Physics game: Discovering the Higgs boson
}

\author{
M. A. M. Souza ${ }^{* 10}$, A. C. S. Nascimento ${ }^{10}$, D. F. Costa ${ }^{2}$, O. Ferreira ${ }^{1}$ \\ ${ }^{1}$ Instituto Federal do Piauí, Parnaíba, PI, Brasil \\ ${ }^{2}$ Universidade Federal do Ceará, Fortaleza, CE, Brasil
}

Recebido em 30 de Abril, 2018. Revisado em 12 de Setembro, 2018. Aceito em 13 de Setembro, 2018.

\begin{abstract}
Neste artigo é proposto a utilização de um jogo didático como complementação metodológica para ensinar Física de altas energias, abordando a temática de Física de Partículas Elementares. A ideia é utilizar estratégias lúdicas e divertidas para ensinar fenômenos estudados no Grande Colisor de Hádrons (LHC), levando os participantes a fazerem novas descobertas didático-científicas, adquirir conhecimento e construírem conceitos que serão utilizados na formulação da teoria do bóson de Higgs e de sua detecção no experimento ATLAS. Embora tais conceitos tenham uma descrição quantitativa extremamente complexa, até certo ponto arraigada a uma análise experimental, é possível torná-la qualitativamente acessível sem precisar recorrer a formulações matemáticas avançadas, conservando, entretanto, seu caráter técnico.
\end{abstract}

Palavras-chave: jogo didático, LHC, bóson de Higgs, ATLAS.

In this article it is proposed the use of a didactic game as a methodological complementation to teach High Energy Physics, addressing the theme of Elementary Particle Physics. The idea is to use playful and fun strategies to teach phenomena studied in the Hadron Large Collider (LHC), leading the participants to make new scientific discoveries, acquire knowledge and construct concepts that will be used in the formulation of the Higgs boson theory and its detection in the ATLAS experiment. Although such concepts have an extremely complex quantitative description, to some extent rooted in experimental analysis, it is possible to make it qualitatively accessible without having to resort to advanced mathematical formulations, while retaining its technical character.

Keywords: didactic games, LHC, Higgs boson, ATLAS.

\section{Introdução}

O processo contemporâneo de ensino e aprendizagem da Física traz elementos da concepção de modelos a partir de objetos concretos, com ênfase em processos empíricos, que remontam à Grécia antiga. Esses métodos de investigação científica, que ainda hoje em dia servem como norteadores no meio científico, ficaram popularizados no século XVI por nomes como Francis Bacon, Willian Gilbert e Galileu Galilei [1].

A evolução da Física como ciência sempre esteve arraigada na construção de experimentos capazes de reproduzir fenômenos naturais e adequar as abstrações dos físicos teóricos à realidade, o que teve como resultado a construção de inúmeros modelos capazes de explicar, por exemplo, a estrutura nuclear [2 4] e as famílias de partículas elementares em um arcabouço teórico chamado Modelo Padrão [5 7].

Com as teorias da Mecânica Quântica Matricial 8 de Werner Heisenberg(1901-1976), a versão ondulatória [9] de Erwin Schrödinger(1887-1961) e a formulação relativística [10] de Paul Dirac(1902-1984), uma descrição teórica mais precisa acerca dos processos nuclea-

*Endereço de correspondência: msouza@ifpi.edu.br res e de interação entre partículas elementares pôde ser obtida, resultando nas teorias mais modernas da Cromodinâmica Quântica [11,12 de David Politzer(1949-), Frank Wilczek(1951-) e David Gross(1941-); na Eletrodinâmica Quântica 13 16 de Richard Feynman(19181988), Sin-Itiro Tomonaga(1906-1979) e Julian Schwinger(1918-1994); e na Teoria eletrofraca $17-19]$ de Steven Weinberg(1933-), Abdus Salam(1926-1996) e Sheldon Glashow(1932-), que unifica a interação eletromagnética com a Teoria de Fermi para a interação fraca mediante a atuação de campos carregados por bósons vetoriais.

Nesse cenário verificou-se que as interações entre as partículas ocorriam através de campos, e cada campo poderia ser representado por uma partícula mediadora, denominada bóson, como pode ser visto na Tabela I. Para interação eletromagnética, temos o fóton como partícula mediadora, a força nuclear forte é mediada pelo glúon e a força nuclear fraca é transmitida pelos bósons $Z^{0}, W^{+}$ e $W^{-}$, sendo que todas estas patículas adquirem suas propriedades de um campo escalar complexo conhecido como campo de Higgs. Em uma Teoria de Grande Unificação, como a Teoria das Supercordas, que quantiza o campo gravitacional, e unifica as quatro interações fundamentais, aparece uma partícula mediadora para força 
Tabela 1: Bósons elementares

\begin{tabular}{lccc}
\hline Nome & Simbolo & Massa $\left(\mathrm{GeV} / c^{2}\right)$ & Descoberta \\
\hline Fóton & $\gamma$ & 0 & 1905 \\
Glúon & $g$ & 0 & 1978 \\
Bósons carregados & $W^{ \pm}$ & 80 & 1983 \\
Bóson neutro & $Z^{0}$ & 91 & 1983 \\
Gráviton & $G$ & 0 & - \\
Bóson de Higgs & $H$ & 126 & 2012 \\
\hline
\end{tabular}

gravitacional, denominada gráviton, que ainda não foi observada diretamente em aceleradores de partículas.

No Ensino Médio, é um grande desafio ensinar Física de Altas Energias, embora as partículas elementares estejam sempre presentes nos conteúdos ministrados, seja para explicar uma ligação química por meio do compartilhamento de elétrons, ou descrever a estrutura atômica e nuclear, onde temos um núcleo formado por prótons e nêutrons rodeado por uma nuvem eletrônica denominada eletrosfera. Noções de radioatividade no estudo da instabilidade nuclear em núcleos radioativos, já fornecem aos estudantes conhecimento de partículas beta, cuja emissão está associada a interação fraca, temos ainda as partículas alfa e a radiação gama, sendo esta última formada pelo quantum da radiação do campo eletromagnético, o fóton. A ideia de campo também está presente no estudo da Eletrostática, do Magnetismo e Gravitação. O campo eletromagnético carregado pelo fóton já pode ser contextualizado no Eletromagnetismo, por meio da transmissão de ondas de rádio, ou mesmo na Ótica ondulatória para explicar os processos de dispersão, onde é possível discutir a dualidade onda-partícula. A própria Teoria da Relatividade abordada no Ensino Médio já fornece subsídios básicos para o estudo de reações entre partículas subatômicas.

Sob esse ponto de vista, justifica-se a importância do resgate de aspectos da teoria quântica de campos e partículas elementares no ensino de Física, os quais muitos autores costumam designar através da tríade pesquisaação-reflexão 20]. Mesmo diante de inúmeros exemplos de sucesso de aplicação dos conhecimentos gerados pela Física, indo de sistemas aplicados às telecomunicações até o uso clínico e diagnóstico de radiações, como a Radioterapia e a Ressonância Magnética Nuclear, muitos estudantes no Ensino Médio adotam uma postura de desinteresse ou mesmo de repulsa em relação à disciplina 21]. Muitos deles a associam a uma ciência complicada que exige extrema dedicação para seu aprendizado. Pode-se dizer que este é um problema constantemente enfrentado por professores da Educação Básica, onde muitos deles alegam que a solução está no estabelecimento das condições adequadas para o desenvolvimento das competências cognitivas dos educandos.

O que se percebe a partir das aulas de Física é que esta disciplina parece alheia à realidade dos alunos, com aulas maçantes, em que predomina uma concepção tradicional de ensino, usando uma metodologia arcaica e nitidamente conteudista. Na maioria das vezes há uma rotina excessiva de aulas expositivas e resolução de exercícios que, em geral, priorizam a memorização de fórmulas matemáticas. Por causa desse procedimento, muitos estudantes são levados a repetir as resoluções de questões similares feitas anteriormente pelo professor, sem que haja, necessariamente, incentivo ao desenvolvimento das habilidades práticas ou cognitivas dos alunos [22].

Diante do que foi exposto, propomos o desenvolvimento de um jogo didático que aborda conhecimentos de Física de Partículas Elementares. Essa proposta faz parte de um conjunto de ações do PIBID, programa governamental de incentivo à atividade docente, cujo foco principal é a formação docente, a melhoria do ensino nas escolas públicas, a divulgação da ciência e a formação de novos cientistas e pesquisadores. O programa proporciona também o primeiro contato entre a maioria dos alunos da licenciatura e a escola. Dessa forma, os futuros professores são submetidos, in loco, às condições reais do trabalho docente. Esta iniciação, para a maioria dos licenciandos, acontece sob a supervisão de um professor de sua instituição formadora e de um professor-supervisor da escola onde está instalado o projeto.

As escolas selecionadas pelo projeto apresentam notas baixas em indicadores como o ENEM (Exame Nacional do Ensino Médio). O principal desafio da atividade foi fazer a transposição didática de tópicos avançados de Física, utilizando uma linguagem acessível ao aluno por meio de um jogo didático, promovendo a conexão entre as áreas de estudo e o meio que os cerca. Todas as imagens utilizadas para construção do tabuleiro do jogo tem licença livre no dominio público da Wikmedia (licença Creative Commons).

Do ponto de vista metodológico e didático, a utilização do jogo visa a complementação de aprendizagem do tema de Física de partículas, uma vez que os participantes adquirem conhecimento enquanto avançam no tabuleiro, cada casa representada por uma partícula ou um experimento do LHC trás uma informação que serve para aprimorar o conhecimento dos estudantes e que pode ser debatida entre os participantes. Busca-se também desenvolver a capacidade cognitiva 23 e de concentração dos alunos, o senso interpretativo e investigativo, pois toda jogada pode levar a uma nova descoberta, além de ter como meta a colaboração científica dentro dos grupos de jogadores.

Nesse contexto, na seção II abordaremos aspectos gerais do Modelo Padrão da Física de partículas, na seção III apresentaremos as regras do jogo, em seguida, na seção IV faremos uma simulação de partida destacando as instruções, penalidades e bonificações. Na seção $\mathrm{V}$ vamos discutir as potencialidades, limitações e aplicabilidade do jogo e as considerações finais serão feitas na seção VI. E por fim temos um Apêndice com o glossário que contém alguns termos técnicos para auxiliar o leitor. 


\section{Interações fundamentais e o bóson de higgs}

A natureza pode ser totalmente compreendida por meio da descrição de quatro interações básicas, responsáveis pelo comportamento dos sistemas físicos da escala subatômica à escala astronômica. Temos a bem conhecida interação gravitacional, cujo o desenvolvimento como ciência pode ser atribuído aos trabalhos de Johannes Kepler (1571-1630) e a teoria da Gravitação Universal de Isaac Newton (1643-1727). Ela é responsável pela dinâmica orbital dos corpos celestes, com efeitos significativos no estudo de objetos massivos do Universo, como buracos negros e estrelas de nêutrons. Atualmente é objeto de estudo da teoria da Relatividade Geral, sendo interpretada geometricamente como resultado da curvatura do espaço-tempo devido a presença de matéria, tendo seus efeitos desprezíveis na escala atômica, o que dificulta a quantização do campo gravitacional.

A interação eletromagnética age nos corpos dotados de carga elétrica, e também atua nos fenômenos magnéticos. Explica as propriedades da matéria assim como as ligações moleculares entre compostos, por meio das forças interatômicas, como as pontes de hidrogênio e a ligação de Van der Waals. É ela que determina o movimento dos elétrons nos orbitais atômicos. Tem inúmeras aplicações no meio tecnológico, sendo responsável pelo funcionamento dos equipamentos de transmissão via satélite, de gravação magnética, equipamentos elétricos e eletrônicos. É utilizada no processamento de sinais elétricos de forma digital e analógica, na Computação Quântica e na Spintrônica.

A interação fraca é responsável pelo decaimento beta. É um processo de transição radioativa entre estados instáveis de alguns núcleos com a emissão de elétrons de alta energia. A teoria inicial do decaimento beta tinha sérios problemas, pois não dava conta do espectro de energia observado experimentalmente e que não podia ser comportado por um único elétron. Em 1930, Wolfgang Pauli (1900-1958) postulou a existência do neutrino, outra partícula que também era emitida no decaimento, sem carga, sem massa e spin $1 / 2$; a existência de uma partícula desprovida de massa, de carga nula e momento de spin 1/2 era necessária para a preservação dos princípios de conservação de energia e momento angular. No interior do núcleo, o decaimento beta pode ser expresso pelas seguintes reações:

$$
\begin{aligned}
& p \longrightarrow n+e^{+}+\nu_{e} \\
& n \longrightarrow p+e^{-}+\bar{\nu}_{e}
\end{aligned}
$$

As equações (1) e 2 mostram o decaimento beta positivo e negativo com a emissão de um pósitron e um neutrino do elétron ou a emissão de um elétron e um antineutrino do elétron, respectivamente. Uma teoria mais precisa só foi proposta em 1934 por Enrico Fermi (1901-1954) 24]. Anos mais tarde essa teoria foi aprimorada com os trabalhos de Richard Feynman (1918-1988) e Murray Gell-Mann (1929-) 25], sendo utilizado na descrição da interação de Fermi um tratamento relativístico coerente a partir da equação de Dirac.

As aplicações do processo de decaimento beta estão presentes em uma classe considerável de fenômenos, que se estendem da fisiologia humana até o desenvolvimento de tecnologia espacial e industrial. Um fenômeno interessante que envolve a emissão beta ocorre no interior do corpo humano, esse processo de radiação é responsável pelo funcionamento do músculo cardíaco 26,27 .

No sangue humano, encontra-se diluído cerca de 30 mg de potássio- $40\left({ }^{40} \mathrm{~K}\right)$, que é um isótopo radioativo do potássio-39, esta concentração é suficiente para emissão de partículas beta. Dentro do coração, existem duas cavidades, chamadas de aurículas direita e esquerda: a primeira tem a função de bombear sangue na circulação pulmonar e a segunda é responsável por distribuir sangue enriquecido com oxigênio por todas as partes do corpo. Ambas são dotadas de células e feixes de fibras nervosas denominadas de nódulo sinoauricular e feixe de Bachman, respectivamente.

O potássio diluído no sangue, dentro dessas cavidades, sofre desintegração por emissão de elétrons e estes, ao colidirem com as terminações nervosas das paredes das cavidades cardíaca, geram um estímulo que faz com que o músculo cardíaco se contraia e se expanda o que faz com que o sangue seja bombeado por todas as partes do corpo.

A Teoria eletrofraca, que unifica o Eletromagnetismo com a interação fraca descreve a emissão beta por meio da transformação de quarks no interior de prótons e nêutrons, com a emissão virtual dos bósons $W^{ \pm}$e $Z^{0}$. Um texto riquíssimo que trata da evolução histórica das teorias de calibre e unificação pode ser encontrado em 28.

A força nuclear forte liga prótons e nêutrons no interior do núcleo atômico e, consequentemente, assegura a estabilidade do núcleo e da matéria hadrônica. É a força de maior intensidade na natureza, daí vem o nome da interação. A primeira teoria da interação hadrônica a descrevia por meio da dispersão nêutron-próton à alta energia, mas esta abordagem não era capaz de caracterizar todo o seu comportamento, de modo que este modelo foi modificado posteriormente. Toda a dinâmica do processo era concebida em termos da energia de ligação do dêuteron, que é um núcleo composto por um próton e um nêutron. O problema era tratado fisicamente com os princípios quânticos de interação entre dois corpos.

Os resultados das experiências de espalhamento entre nucleons levam às seguintes propriedades da interação nucleônica: a propriedade de saturação, isto é, um único nucleon só pode interagir com um número limitado de outros nucleons; a independência da carga, ou seja, as forças nucleares são simétricas em relação à carga, a intensidade de interação numa reação (nn) é idêntica a uma reação (pp), e a presença de forças de troca. 
Na Mecânica Quântica, quando duas partículas interagem mutuamente, existe sempre um estado em que alguma propriedade pode ser compartilhada, produzindo uma interação de troca para os nucleons, Werner Heisenberg (1901-1976) supôs que essa propriedade seria a carga [29]. A propriedade de força de troca está ligada intimamente à teoria mesônica das forças nucleares [30] proposta pelo físico japonês Hideki Yukawa (1907-1981), em 1935, que em analogia à interação eletromagnética mediada pela troca de fótons, supôs que a interação hadrônica entre nucleons fosse resultado da troca de um méson $\pi$; o fato dessa partícula possuir massa possibilitava caracterizar essa interação como sendo de curto alcance, além disso, os mésons possuem carga $\left(\pi^{-}, \pi^{+}\right)$ou podem ser eletricamente neutros $\left(\pi^{0}\right)$, requisito necessário para que, em uma interação, a carga das partículas envolvidas seja permutada. Nesse processo, explicitamente, um próton pode converter-se em um nêutron e vice-versa.

Hoje em dia, a teoria mais moderna da força nuclear forte, a Cromodinâmica Quântica, foca-se na ligação entre os constituintes de prótons e nêutrons, denominados de quarks 31,32, atribuindo a estes últimos a propriedade de carga de cor e de sabor, sendo o glúon a partícula responsável por esta interação. A teoria de Yukawa da interação por píons é conhecida atualmente como efeito residual, e ainda é uma abordagem efetiva para a Física Nuclear 33.

A maior aplicação da força nuclear forte ocorre nos reatores de fissão nuclear utilizados para produzir energia elétrica, onde o urânio, ao ser bombardeado por nêutrons, absorve essas partículas e torna-se instável, sofrendo uma bipartição (gerando dois novos núcleos mais leves). A energia correspondente à força nuclear forte que unia os prótons e nêutrons no núcleo de urânio é liberada, de forma simplificada, na forma de energia cinética dos dois núcleos residuais, energia esta que pode ser aproveitada dentro do reator de uma usina nuclear ou pode ser usada para fins bélicos, como no caso da bomba atômica. No processo de bipartição do núcleo ocorre também a emissão de nêutrons que irão alimentar a reação em cadeia dentro do reator ou em uma explosão nuclear.

A descrição da interação eletrofraca com a interação nuclear forte em um único arcabouço teórico resultou no conhecido Modelo Padrão da Física de Partículas elementares, que agrupa todas as partículas e suas antipartículas em três categorias: léptons, quarks e bósons de calibre, como pode ser visto de forma resumida na Figura 1. E eis aqui o ponto chave de toda a nossa discussão, pois para que todo o modelo funcione é necessário um campo, responsável por atribuir às famílias de partículas elementares as propriedades de massa tal qual as conhecemos, trata-se de um campo escalar fundamental, representado pelo bóson de Higgs, descoberto em julho de 2012 no LHC (Large Hadron Collider - Grande Colisor de Hádrons), vindo estes resultados a serem corroborados no início de 2013; o que valeu o Prêmio Nobel de Física

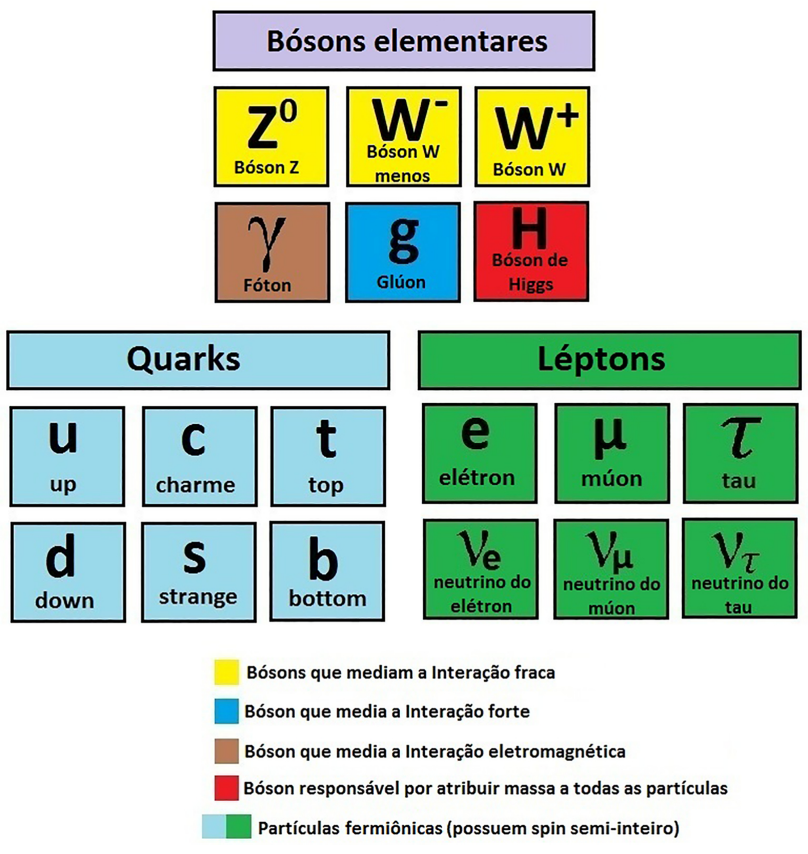

Figura 1: Modelo Padrão da Física de Partículas, onde temos representados os léptons, quarks e os bósons mediadores de interação.

para Peter Higgs (1929-) e François Englert (1932-), que previram teoricamente a existência do bóson em 1964.

Campos escalares podem ser definidos como uma função da posição de um ponto do espaço, independente do sistema de coordenadas adotado, sejam as coordenadas definidas no espaço euclidiano ou no espaço-tempo de Minkowski. Não importando a métrica do espaço-tempo adotada, são invariantes relativísticos, pois possuem o mesmo valor quando mensurados por observadores distintos em referenciais separados e trata-se de um tensor de ordem zero. Na Física Clássica são comumente empregados para representar distribuição de temperatura em um meio ou o campo de pressões em um fluido. Também estão associados a energia potencial de um campo de forças, como o potencial gravitacional ou o potencial elétrico na Eletrostática.

Na Física de altas energias, eles ainda podem ser reais ou complexos, o campo de Higgs, por exemplo, é representado por um campo escalar complexo, ao passo que na Cosmologia e Matéria Condensada é mais comum empregarmos campos escalares reais. Na Teoria de campos defini-se campo escalar real como sendo uma função real do quadrivetor $\chi^{\mu}=(t, \vec{r})$ no espaço-tempo de Minkowski, isto é, $\phi=\phi\left(\chi^{\mu}\right)$. Campos escalares complexos também são utilizados para representar partículas carregadas que decaem por meio da interação fraca, como os mésons.

O campo de Higgs permeia todo o espaço comportandose como um fluido de viscosidade não nula, ou seja, as partículas que interagem com esse campo sofrem uma resistência natural ao seu movimento, prefigurando aquilo 
que entendemos ser a massa ou inércia. Quanto maior a massa da partícula, mais resistência ela enfrenta, daí o fato de algumas interações terem seu alcance reduzido, pois são mediadas por bósons massivos. Em contrapartida, partículas não massivas podem deslocar-se no vácuo com a velocidade da luz. O bóson de Higgs surge da quantização do campo de Higgs, isto é, é o estado de excitação de energia do campo 34].

Na Física de Partículas, o campo de Higgs, cujo estado de vácuo é constante com valor igual a $246 \mathrm{GeV}$, adquire sua própria massa do mecanismo de quebra espontânea de simetria do vácuo 35 36, como ilustrado na Figura 2 , em que temos representado o potencial de um campo escalar $\phi$, onde o mínimo do potencial representa o estado de vácuo. De forma geral, podemos dizer que um sistema é simétrico se o mesmo não muda suas características e/ou propriedades frente a alterações dos parâmetros que o descrevem. Na Teoria de campos, dizemos que uma teoria descrita por uma densidade de Lagrangeana $\mathcal{L}(\phi)$, onde $\phi$ representa um campo escalar, é simétrica se esta torna-se invariante frente à transformação:

$$
\mathcal{L}(\phi) \rightarrow \mathcal{L}(-\phi),
$$

o estado de vácuo é degenerado e os estados possíveis transformam-se um no outro em detrimento dessa simetria.

Em Física, a aplicação mais importante de simetria está no teorema de Noether [37, que estabelece que para cada simetria contínua em um sistema físico existe uma correspondente lei de conservação. Assim, pode-se mostrar que a conservação de momento linear está associada à simetria de translação ou paridade do sistema. A conservação de energia associa-se à uniformidade temporal e a conservação de carga elétrica a uma simetria de calibre, entre outras. Um sistema possui sua simetria quebrada quando as alterações promovidas neste, modificam sua estrutura, levando ao aparecimento de fases distintas. Fenômenos como esse aparecem em processos de transição de fase em sistemas de Matéria Condensada, como em cadeias poliméricas de poliacetileno 38. Também podemos encontrar quebra de simetria em Modelos cosmológicos. Essa quebra de simetria seria responsável pelo surgimento de defeitos topológicos no Universo
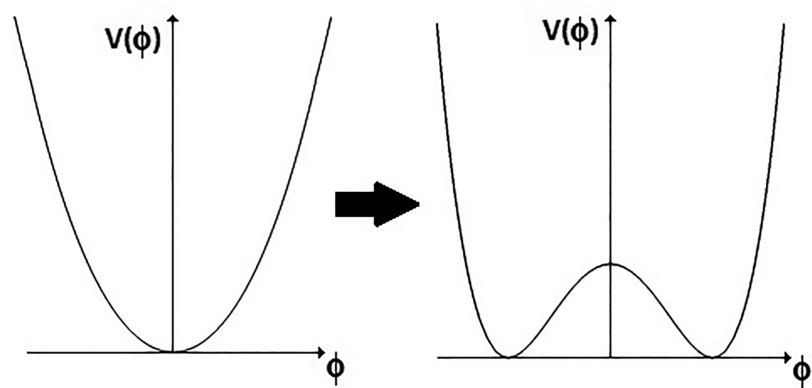

Figura 2: Representação do mecanismo de quebra espontânea de simetria do vácuo para um campo escalar. primordial que seriam cordas cósmicas [39], os monopolos magnéticos 40,41, introduzidos por Gerardus 't Hooft (1946-) e Alexander Polyakov, e ainda defeitos denominados de texturas 42,43 .

Em Teoria de Campos, um dos mecanismos de quebra de simetria de grande interesse é aquele que promove a geração de massa para o campo.

No gráfico da esquerda na Figura 2, vemos claramente que o estado de energia mais baixo está na origem, já no segundo gráfico, onde a simetria foi quebrada, temos dois estados de vácuo onde o campo adquire massa, ou seja, o estado de menor energia não é mais nulo. A existência de um estado de vácuo diferente de zero também quebra espontaneamente a simetria de calibre da interação eletrofraca, cujo mecanismo gera massa para os bósons de calibre $Z^{0}, W^{+}$e $W^{-}$, mediadores dessa interação.

Existem ainda outros mecanismos desse tipo, por exemplo, para um sistema descrito por dois campos escalares $\phi_{1}$ e $\phi_{2}$, a quebra espontânea de simetria de um grupo contínuo de simetria, como o grupo de rotações $\mathrm{SO}(2)$, leva ao surgimento de massa para um dos dois campos, enquanto o outro permanece sem massa. Esse campo sem massa é denominado de Bóson de Goldstone 44,45$]$.

\section{Regras}

O objetivo deste jogo, intitulado "Descobrindo o bóson de Higgs" é seguir pelos experimentos do Grande Colisor de Hádrons (LHC), construído na cidade de Genebra na Suíça, iniciando pelo experimento $\mathrm{LHCb}$ até a descoberta do bóson de Higgs no experimento ATLAS, ver Figura 3. Durante a jornada, os jogadores irão entrar no mundo das partículas elementares, deverão enfrentar obstáculos, responder perguntas sobre o tema, encarar desafios como escapar de buracos negros e de um loop temporal, evitar a radiação gama, e de serem confinados, pela força nuclear forte, no plasma de quarks e glúons.

Mas nem só de obstáculos é feito este jogo. Participantes astutos poderão encurtar distâncias com viagens no espaço-tempo, poderão ter a ajuda de neutrinos e prótons para avançar. Utilizar o decaimento dos píons para escapar da zona do buraco negro e podem chegar à

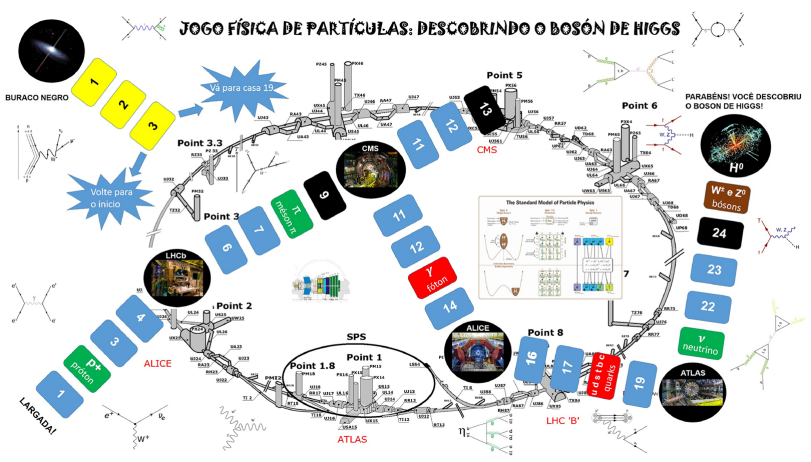

Figura 3: Modelo de tabuleiro para o jogo. 
grande descoberta com a ajuda dos bósons W e Z. No tabuleiro de jogo existem três tipos de casas, conforme descrição a seguir.

1. PARTícULAS: Ao cair em uma casa que possui o símbolo de uma partícula subatômica o participante lê a informação e segue as instruções: avançar, retornar, jogar de novo ou ficar uma rodada sem jogar.

2. EXPERIMENTOS DO LHC: Ao cair em um experimento do LHC o jogador deverá ler a informação e seguir as instruções: ficar uma rodada sem jogar, avançar para zona do buraco negro ou escolher um jogador para responder uma pergunta.

3. BURACO NEGRO: As zonas dos buracos negros estão representadas pelas casas pretas e ao cair nessas casas o jogador será sugado até o buraco negro. Para afastar-se do horizonte de eventos, o participante deverá percorrer as casas amarelas respondendo perguntas. Na terceira casa, se o jogador acertar a pergunta irá automaticamente para casa 19, caso erre deverá retornar ao início do jogo.

\section{PEÇAS DO JOGO:}

- 1 Tabuleiro;

- 1 dado;

- Cartas com as perguntas;

- 4 cones de cores diferentes para representar os jogadores ou grupos de jogadores.

Para decidir qual participante inicia o jogo, todos devem jogar o dado e quem tirar a maior pontuação terá o direito de ser o primeiro a dar a largada. Em caso de empate, todos deverão jogar o dado novamente. O primeiro participante escolhe uma carta e terá de responder uma pergunta (ver Figura 4), caso acerte, ele joga o dado e avança no tabuleiro, caso erre a pergunta, permanece onde está e passa a vez e assim sucessivamente para todos os jogadores ao longo do jogo.

O jogo é indicado para estudantes do Ensino Médio, alunos do curso de graduação em Física, assim como também pode ser utilizado em cursos de qualificiação e formação de professores. Todas as respostas estão contidas no manual do jogo. O jogo completo, disponível de forma gratuita, pode ser acessado em [46].

\section{Instruções, Penalidades e Bonificações. Vamos jogar!}

A seguir apresentamos as penalidades e bonificações do jogo ao cair em uma determinada casa, destacando que a medida que o participante avança ele adiquire mais informações e conhecimentos a respeito das partículas elementares e dos experimentos realizados no LHC. Vamos simular uma partida hipotética, ideal e rápida, na qual o jogador irá usufruir de todas as bonificações que

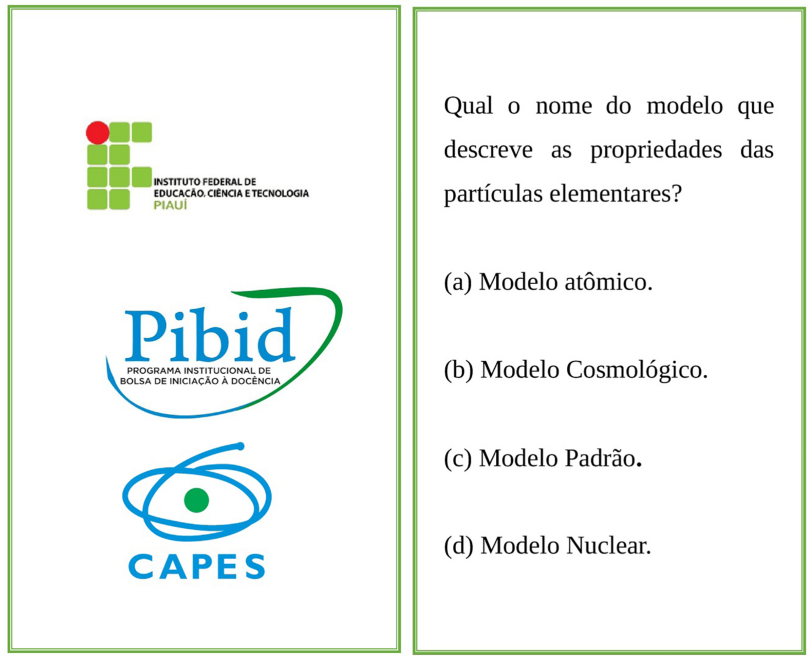

Figura 4: Frente e verso do modelo de carta com perguntas e opções de respostas.

o jogo oferece, bem como sofrerá todas as penalidades possíveis até alcançar a grande descoberta.

No início, após respondida uma pergunta do baralho de cartas de forma correta, o jogador lança o dado. E no primeiro lançamento o jogador obtém o duque, ou seja, avança duas casas e chega até o próton (ver Figura 5). O jogador obtem a informação a respeito da partícula e joga mais uma vez conforme as instruções.

PRÓTON: Os prótons são partículas que compõe o núcleo atômico, possuem carga positiva e massa de $1,67 \times$ $10^{-27} \mathrm{~kg}$. Pertencem à família dos hadróns, possuindo uma estrutura interna formada por dois quarks up e um quark down.

Os feixes de prótons dentro do LHC podem colidir a uma energia de até $13 \mathrm{TeV}$, ou seja, cada feixe que circula em direções opostas pode alcançar uma velocidade de $99 \%$ da velocidade da luz $\left(c=3 \times 10^{8} \mathrm{~m} / \mathrm{s}\right)$ antes da colisão. Você está pegando carona em um feixe extremamente energético. Jogue mais uma vez.

No segundo lançamento, ele obtém o terno e avança três casas até o experimento LHCb. Após escolher um participante para responder uma pergunta, ele passa a vez e aguarda a próxima rodada.

EXPERIMENTO LHCb: A sigla significa Large $H a$ dron Collider beauty. Este experimento tem o objetivo de detectar violações na simetria $\mathrm{CP}$ (Carga Paridade) no decaimento de mésons b, conjunto formado pelos quarks
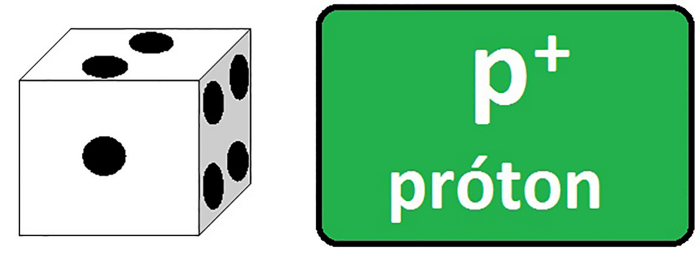

Figura 5: O jogador avança duas casas e chega até o próton. 
bottom e anti-bottom. Decaimentos de mésons b são muito raros 47]. Escolha um participante para responder uma pergunta e aguarde sua vez de jogar.

$\mathrm{Na}$ rodada seguinte, após responder novamente uma pergunta, é lançado o dado, e o jogador no seu terceiro lançamento obtém novamente o terno e cai na casa do méson (ver Figura 6).

Em um acelerador de partículas, quando um antipróton colide com um próton estacionário ocorre a produção de um grande número de partículas, entre elas um méson $\pi^{-}$, que decai segundo a reação $\pi^{-} \rightarrow \mu^{-}+\bar{\nu}_{\mu}$, onde temos como subproduto um múon e o antineutrino do múon. Em seguida temos ainda o decaimento do múon segundo a reação $\mu^{-} \rightarrow e^{-}+\bar{\nu}_{e}+\nu_{\mu}$, onde temos um elétron, um antineutrino do elétron e o neutrino do múon, respectivamente. Na primeira reação temos conservação de carga elétrica, pois $-1=-1+0 \Rightarrow-1=-1$, o zero está associado ao neutrino que não possui carga elétrica. Na segunda reação podemos ver que ocorre a conservação de carga elétrica e do número leptônico, que é um número quântico associado aos léptons em uma reação entre partículas elementares. Léptons são partículas leves, como os elétrons, neutrinos e múons, que interagem por meio da força nuclear fraca. É atribuído o valor de +1 para o número leptônico dos léptons e -1 para as antipartículas dos léptons. Equacionando temos $+1=+1-1+1 \Rightarrow+1=+1$.

MÉSON $\pi$ (PÍON): Mésons são partículas que pertencem à família dos hádrons, são formados por quarks e antiquarks. O píon é responsável pela interação prótonnêutron no interior nuclear, é extremamente instável decaindo no múon. Aproveite a instabilidade do píon e pegue carona com o múon, fugindo da zona do buraco negro, até o experimento CMS.

EXPERIMENTO CMS: Em português a sigla significa Solenoide de Múon Compacto, é um dos pontos onde ocorrerá a colisão entre os feixes de prótons, alguns de seus objetivos são detectar dimensões extras, estudar a colisão entre íons pesados e investigar a Física na faixa de energia de TeV [47]. Parabéns, até aqui você se saiu bem! Você tem uma importante escolha a fazer: caso seja destemido, poderá aventurar-se e desvendar os mistérios de um buraco negro na próxima rodada; se for cauteloso, espere a sua vez de jogar e avance na direção do experimento ALICE.
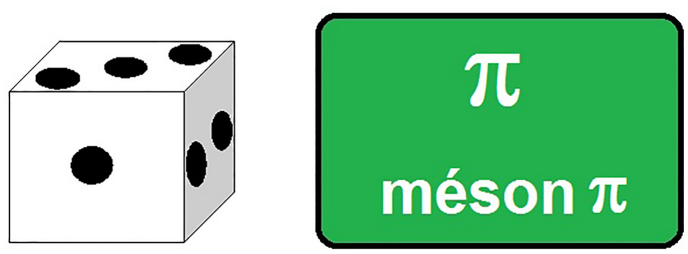

Figura 6: O jogador avança três casas e chega até o méson.
Nesse primeiro momento o participante irá ignorar o buraco negro, afinal as perguntas nessa jornada são mais difíceis, mas ele ainda não está salvo da singularidade, vamos seguir na direção do experimento ALICE. Respondendo uma pergunta corretamente e no seu quarto lançamento do dado, nosso explorador obtém novamente o terno e cai na casa do fóton (ver Figura 7) e deve retornar duas casas até a casa 11.

FÓTON: São partículas que formam a radiação eletromagnética em todo o espectro, como a luz visível, os raios X, a radiação ultravioleta, o espectro infravermelho e a radiação gama. Cuidado! A radiação gama de alta energia gerada nas reações e decaimentos de partículas no LHC pode ser perigosa. Retorne 2 casas antes que você seja atingido!

No seu quinto lançamento, após responder mais uma pergunta, o jogador obtém a quadra e vai até o experimento ALICE. Escolhe um participante para responder uma questão e passa a vez.

EXPERIMENTO ALICE: Em Português a sigla significa Um Grande Experimento Colisor de Íons, tem o objetivo de estudar o plasma de quarks e glúons obtido pela colisão entre íons de altas energias. Cuidado, você está entrando nos domínios da força nuclear forte, seja cauteloso ou poderá ficar confinado no plasma [47]. Aguarde sua vez de jogar e escolha um participante para responder uma pergunta.

Na próxima rodada, o jogador retira uma carta do baralho e responde a pergunta, tendo êxito em sua resposta, obtém em seu sexto lançamento o terno, e cai na casa dos quarks (ver Figura 8). Os quarks possuem a carga de cor na Cromodinâmica Quântica. É similar a um número quântico, e explica porque os quarks, que são férmions e obedecem ao princípio de exclusão de Pauli, ligam-se para formar prótons e nêutrons.

Os quarks podem ter três cores, azul, vermelho e verde, e três respectivas anticores, de modo que a união entre
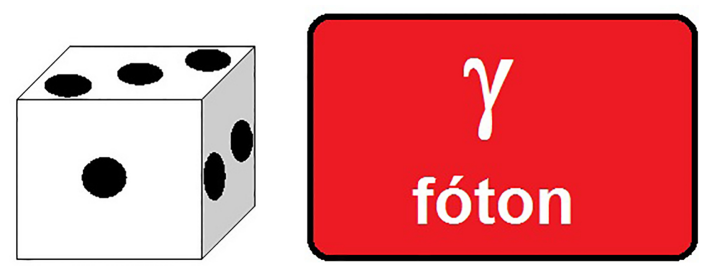

Figura 7: $O$ jogador avança novamente três casas e chega até o fóton.
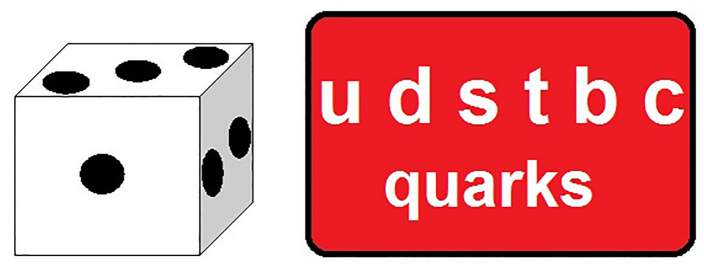

Figura 8: O jogador avança até a casa dos quarks. 
três quarks de cores diferentes ou uma cor e sua anticor geram a neutralidade de carga de cor [48].

Os quarks são distinguidos pelo sabor, que é um número quântico que diferencia os tipos de quarks e léptons, que seriam indistinguíveis em relação às propriedades de carga, spin, etc. Para os léptons temos os sabores elétron $(\mathrm{e}), \operatorname{muon}(\mu), \operatorname{tau}(\mathrm{t})$, neutrino do elétron $\left(\nu_{e}\right)$, neutrino do muon $\left(\nu_{\mu}\right)$ e neutrino do $\operatorname{tau}\left(\nu_{t}\right)$.

QUARKS: São partículas elementares que compõe os hádrons, como prótons e nêutrons. Podem ser de seis tipos ou sabores: $u p(\mathrm{u})$, down $(\mathrm{d})$, strange(s), charm(s), bottom(b) e top(t). Os quarks são confinados nos hádrons pela força nuclear forte, a mais intensa das interações na natureza. Parece que você não está com sorte, não há como fugir da força forte, você está confinado. Fique duas rodadas sem jogar.

Após ficar duas rodadas sem jogar, nosso explorador retira uma carta do baralho e acerta a pergunta proposta, de modo que no seu sétimo lançamento do dado ele tira um duque, e vai para o experimento ATLAS.

EXPERIMENTO ATLAS: A sigla ATLAS em português significa Dispositivo Instrumental Toroidal para o LHC. É um detector de partículas que tem o objetivo de encontar o bóson de Higgs e partículas supersimétricas 47]. Você está perto de fazer uma grande descoberta científica. Explore mais este equipamento e desvende sua estrutura. Fique uma rodada sem jogar!

Passada uma rodada, o jogador responde mais uma pergunta e em seguida lança o dado pela oitava vez, obtendo um ás e caindo na casa do neutrino, conforme Figura 9 .

NEUTRINO: Neutrinos são partículas elementares que pertencem à família dos léptons, é extremamente leve e não possui carga elétrica.

O neutrino possui uma seção de choque muito pequena de modo que praticamente não interage com a matéria, por isso também é chamada de partícula fantasma, cerca de 65 bilhões de neutrinos atravessam uma área de $1 \mathrm{~cm}^{2}$ da superfície da terra por segundo. Eles são formados no Sol por meio das reações nucleares.

O jogo virou, pegue carona com o neutrino, não interaja com nada e avance duas casas!

O participante, utilizando a bonificação, avança até a casa 23. Passa a vez de jogar e na próxima rodada retira uma carta do baralho, responde a pergunta, lança o dado pela nona vez e obtém novamente o ás, caindo na casa 24, a casa do buraco negro!!!
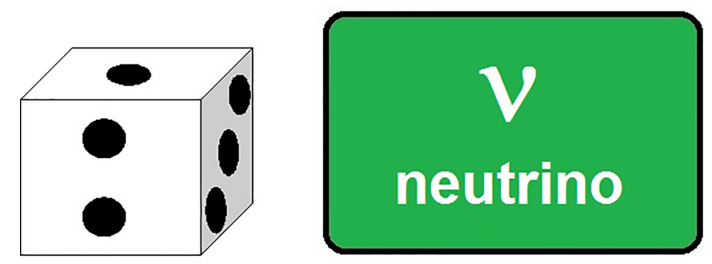

Figura 9: O jogador avança até a casa do neutrino.
Ele é transportado para a singularidade e terá que responder uma série de três perguntas, ver Figura 10. sem lançar o dado, para escapar da atração gravitacional do horizonte de eventos.

BURACO NEGRO: É uma singularidade do espaçotempo originada do colapso de uma estrela supermassiva com limite superior a 8 massas solares, que ao extinguirse cria uma região no espaço extremamente densa com um campo gravitacional tão intenso que nem a luz consegue escapar de seu interior, por isso recebem esse nome. Em certas condições, eles supostamente podem permitir viagens no espaço-tempo. Fique tranquilo, pois no LHC isso é apenas uma especulação e mesmo que viessem a ser criados, teriam dimensões milhões de vezes menor que 1 micrometro, com um tempo de vida de $10^{-27}$ segundos, não representando nenhuma ameaça ao nosso planeta. Vá para o buraco negro e aguarde a sua vez de jogar. Caso seja bem sucedido nas suas respostas você escapará da atração gravitacional e poderá avançar no espaço tempo! Caso falhe, cairá em um loop temporal e retornará para o início do jogo!

Caso o jogador chegue na terceira casa da zona do buraco negro e erre a pergunta, ele automaticamente retorna à primeira casa do jogo. Mas nosso explorador é bem sucedido em suas respostas e consegue escapar do buraco negro, avançando até a casa 19. Na rodada seguinte, respondendo de forma acertiva a uma nova pergunta, o jogador lança o dado pela décima vez e obtém a sena, indo parar na casa dos bósons $\mathrm{W}$ e Z (ver Figura 11.

BÓSON W $\mathbf{W}^{ \pm} \mathbf{E} \mathbf{Z}$ : São partículas que mediam a força nuclear fraca. O bóson $\mathrm{W}$ possui carga elétrica, representado como $W^{+}$e sua antipartícula é dada por $W^{-}$, são responsáveis pela emissão e absorção de neutrinos. O bóson $\mathrm{Z}$ é neutro, sendo ele mesmo a sua antipartícula, é responsável pela transferência de momento, spin

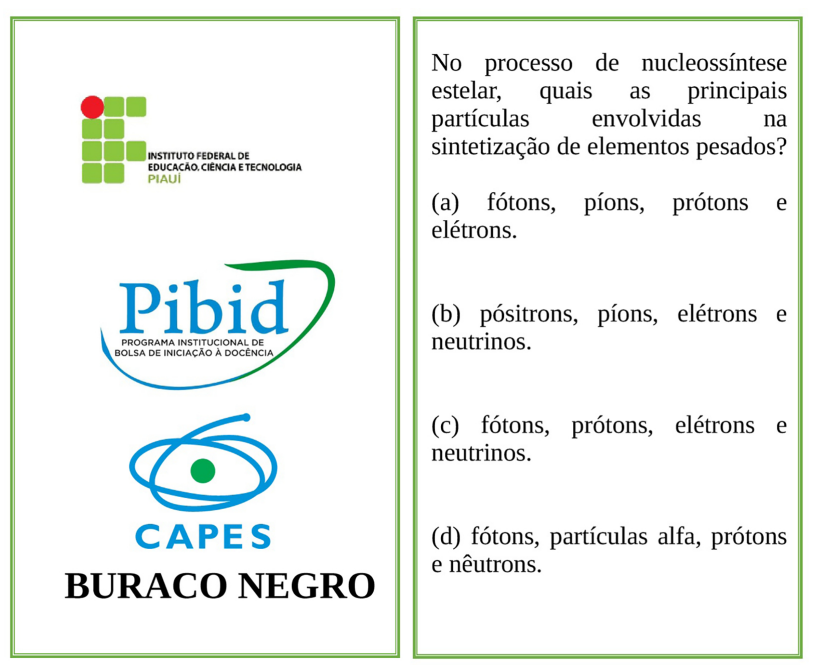

Figura 10: Frente e verso do modelo de carta com perguntas para a zona do buraco negro. 


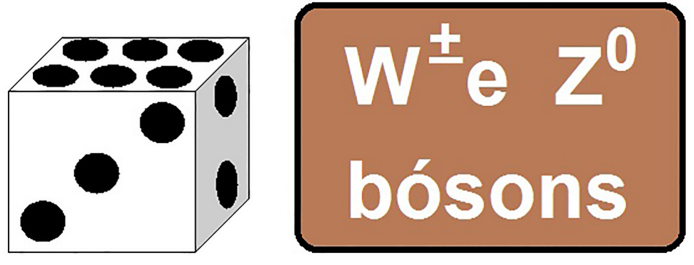

Figura 11: $\mathrm{O}$ jogador avança até a casa dos bósons $W^{ \pm}$e Z .

e energia nas interações de espalhamento de neutrinos por bárions. Os bósons $\mathrm{W}$ e Z estão presentes como um subproduto da construção do campo de Higgs [49].

Você está quase lá, há um passo de descobrir o bóson de Higgs. Responda uma pergunta extra e avance na direção da grande descoberta!

Os bósons $W^{ \pm}$e Z mediam a interação fraca, responsável pelo decaimento beta, que é um processo de transição radioativa entre estados instáveis de alguns núcleos com a emissão de elétrons de alta energia. No interior do núcleo, o decaimento beta pode ser expresso pelas seguintes reações: $p \longrightarrow n+e^{+}+\nu_{e}$ e $n \longrightarrow p+e^{-}+\bar{\nu}_{e}$. Que mostram o decaimento beta positivo e negativo com a emissão de um pósitron e um neutrino do elétron ou a emissão de um elétron e um antineutrino do elétron, respectivamente.

Em ambas as reações ocorre conservação de carga elétrica, no decaimento beta positivo: $+1=0+1+0 \rightarrow$ $+1=+1$, onde estamos considerando a carga elétrica do nêutron nula; no decaimento beta negativo: $0=+1-$ $1+0 \rightarrow 0=0$. A Teoria Eletrofraca descreve a emissão beta por meio da transformação de quarks no inteior de prótons e nêutrons, com a emissão virtual dos bósons $W^{ \pm}$e $Z^{0}$.

Nesta etapa, o jogador responde mais uma pergunta, se acertar, joga novamente o dado e avança com qualquer resultado para casa final, caso erre, permanece onde está. Vamos supor que nosso explorador acertou a última pergunta e tem o direito de lançar o dado pela décima primeira e última vez.

Em seu lançamento ele obtém a quina e avança de forma impetuosa na direção da grande descoberta, desvendendo um dos maiores mistérios da Física, o mecanismo de geração de massa para todas as partículas elementares, um campo escalar complexo carregado pelo bóson de Higgs (ver Figura 12. Você venceu!!

BÓSON DE HIGGS: O bóson de Higgs é a peça chave do Modelo Padrão da Física de partículas, carrega o campo responsável pela geração de massa de todas as partículas conhecidas. Possui spin nulo e sua massa é proveniente do mecanismo de quebra de simetria do vácuo 7. 50,51 e tem valor $125.3 \pm 0.6 \mathrm{GeV} / \mathrm{c}^{2} \approx 126 \mathrm{GeV} / \mathrm{c}^{2}$. Em Julho de 2012 foi anunciado que uma partícula com as mesmas características do bóson de Higgs foi descoberta nos Experimentos ATLAS e CMS, vindo estes resultados a serem corroborados no início de 2013, o que valeu o Prêmio Nobel de Física para Peter Higgs e François

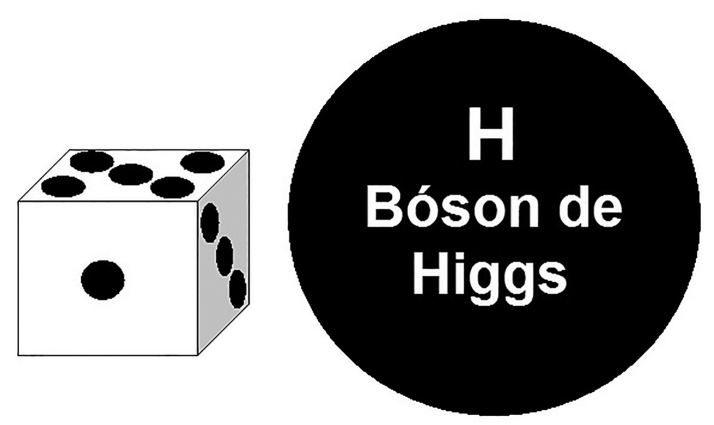

Figura 12: O jogador avança até a casa do bóson de Higgs e faz a grande descoberta.

Englert, que previram teoricamente a existência do bóson em 1964 !

PARABÉNS VOCÊ ACABA DE FAZER UMA GRANDE DESCOBERTA E REVOLUCIONAR A FÍSICA, SINTA-SE ORGULHOSO DE ENTRAR PARA HISTÓRIA DA CIÊNCIA E CONTRIBUIR PARA A COMPREENSÃO DO UNIVERSO E DESENVOLVIMENTO DA HUMANIDADE!

\section{Discussão do jogo}

O Programa Institucional de Bolsas de Iniciação a Docência (PIBID) tem como principais objetivos o desenvolvimento da responsabilidade social e política da docência, articulação entre teoria e prática, valorizando o exercício docente, e a enxergar a pesquisa como princípio de formação, estimulando o uso das novas tecnologias de informação e comunicação nos processos de ensinar e aprender de forma a estabelecer ligações entre áreas do conhecimento. E já propiciou o desenvolvimento de outros trabalhos na área de Ensino de Física 52 54]. As Principais ações do programa são:

- Demonstrações de experimentos Físicos com materiais de baixo custo e fácil aquisição, que proporcionem aos estudantes a comprovação da teoria abordada em sala de aula;

- Realização de jogos, brincadeiras e gincanas com temáticas da disciplina de Física, que despertem a curiosidade dos estudantes e permitam "aprender brincando";

- Escolha de temas transversais a serem abordados através de oficinas, dramatização, paródias, exposição de feiras, seminários e palestras.

Dentro do segundo item, foi idealizado este jogo que visa inserir os estudantes em uma nova temática, pouco usual no currículo das escolas, que é a Física de partículas elementares. É uma oportunidade de apresentar ao aluno, através das explicações das perguntas feitas durante o jogo, a estrutura e funcionamento de uma acelerador de partículas e o quão importante é a ciência desenvolvida 
nesse meio e suas contribuições para o mundo moderno, seja no entendimento da constituição da natureza, ou mesmo na tecnologia gerada e que pode ser utilizada para fins de diagnóstico e tratamento médico. A aplicação do jogo nas escolas públicas da rede estadual de Ensino pode ser vista nas Figuras 13 e 14

O aluno de Ensino Médio ou do Ensino Supeior a priore não teria muita dificuldade de entender a dinâmica de reação de partículas elementares, uma vez que, ao adentrar neste assunto, alguns conteúdos que servem de pré-requisitos para tal entendimento já foram vistos, em parte nas disciplinas de Química, como o conceito de átomo, spin, íons, energia de ligação, massa e número atômico, etc., e também na própria disciplina de Física, nos tópicos relacionados a Eletromagnetismo, Relatividade Restrita e Mecânica Quântica.

O aluno que estudou Reações Químicas também não teria dificuldades de entender como se processa uma reação, uma vez que a notação empregada para descrever ambas é idêntica. Por outro lado, é importante que o professor, como mediador do conhecimento, seja capaz de contextualizar os conteúdos ministrados com o cotidiano do aluno; todos os processos na escala subatômica são passíveis de uma aplicação prática e podem ser estendidos a outras áreas do saber.

Trata-se de um esforço na busca de esclarecer fenômenos de grande importância científica e utilidade prática no mundo moderno, o que está de acordo com a proposta de Terrazzan [55], que defende a atualização do currículo

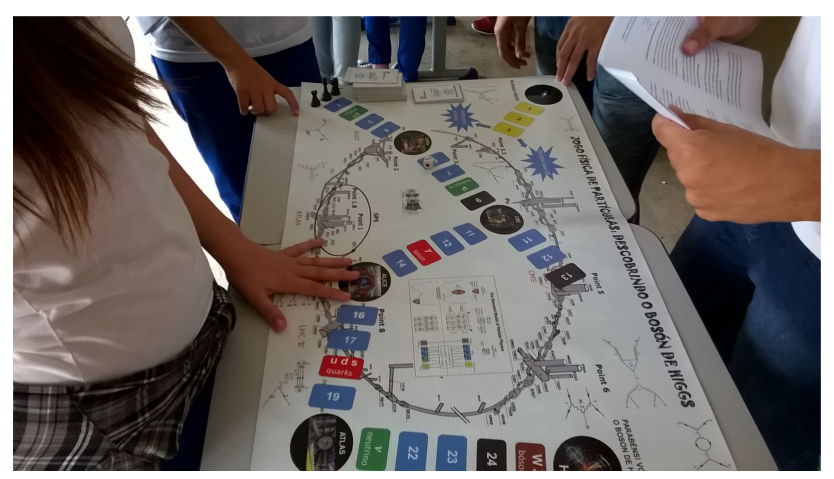

Figura 13: Alunos brincando com o jogo.

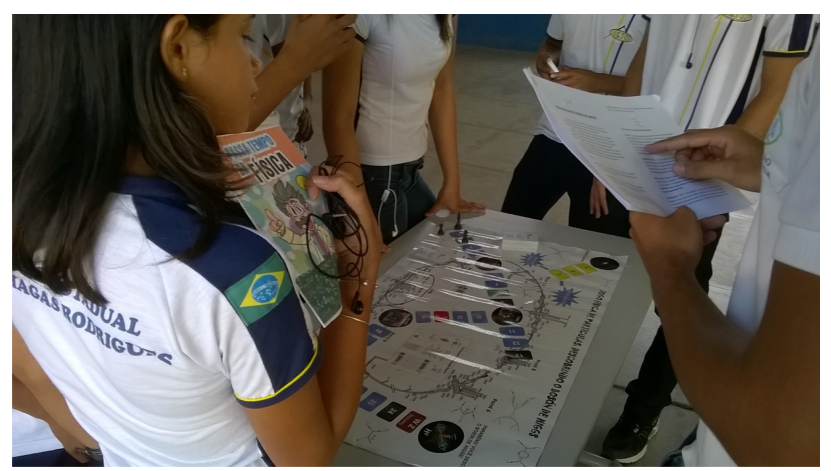

Figura 14: Alunos brincando com o jogo em uma outra escola. de Física doravante o desenvolvimento da ciência contemporânea como necessidade de criar cidadãos conscientes capazes de transformar a realidade, e que ainda foi enfatizado por Aubrecht [56] na conferência sobre ensino de Física Moderna em Abril de 1986 no FERMILAB (Fermi National Accelerator Laboratory), Batavia, Illinois, onde foi defendida a inclusão de tópicos de pesquisa em Física no Ensino Médio. Um texto interessante sobre revisão das literaturas que tratam do tema Física Moderna e Contemporânea no Ensino Médio pode ser encontrado em 57.

Outro aspecto que pode ser explorado dentro da fenomenologia de partículas elementares é a utilização de recursos computacionais, como simulações em java, no estudo experimental acerca da produção de energia nuclear, fissão e fusão nuclear, decaimento radioativo, espalhamento de partículas nucleares, etc. Existem na internet muitos softwares gratuitos com ambientes gráficos interativos e de fácil operação [58]. Esse é um ponto-chave, uma vez que o processo ensino aprendizagem tornar-se-á significativo se o aluno materializar os conceitos abstratos da Física teórica, conectando a fenomenologia dos processos físicos à sua vida prática mediante a observação e interação direta com um laboratório virtual.

A utilização de jogos didáticos em sala de aula, contribui principalmente para o desenvolvimento cognitivo, através da atividade investigativa, e para a contextualização sócio-cultural dos estudantes. Esta atividade, assim como simulações ou modelagens computacionais, facilitam a construção de relações e significados, favorecendo a aprendizagem construtivista $59 \sqrt[61]{6}$, podendo ainda:

1. Elevar o nível do processo cognitivo, exigindo que os estudantes pensem num nível mais elevado, generalizando conceitos e relações;

2. Exigir que os estudantes definam suas ideias mais precisamente;

3. Propiciar oportunidades para que os estudantes testem seus próprios modelos cognitivos, detectem e corrijam inconsistências.

O jogo segue um sistema de cores intuitivo e bem familiar aos estudantes, as casas verdes representam bonificações, significa avance. As casas vermelhas estão associadas a penalidades, pare e fique sem jogar. O amarelo nas casas da zona do buraco negro significa atenção, seja cauteloso.

Embora o bóson de Higgs tenha sido detectado nos experimentos ATLAS e CMS, optamos por dar destaque a sua descoberta somente no Experimento ATLAS, devido ao caráter linear do jogo, que se desenvolve segundo uma dinâmica construtivista e simula um jogo RPG(os jogadores representam cientistas/exploradores em busca de uma grande descoberta cientifica) com um único final. A escolha também foi norteada pelo fato do ATLAS ser o maior detector de partículas do LHC e que têm como principal finalidade observar o bóson de Higgs. 
Apesar da expectativa inicial positiva quanto a aplicação do jogo no Ensino Médio, podemos notar na prática algumas dificuldades na aplicação do mesmo para estudantes do Ensino Médio das escolas públicas brasileiras, seja pela pouca familiaridade com o tema abordado, mesmo já conhecendo algumas partículas, seja por uma barreira cognitiva desenvolvida pelos estudantes, que já estão habituados a aulas tradicionais sem contextualização com o meio cientifico e tecnológico, e sempre atribuem à disciplina de Física o adjetivo de algo complexo e de difícil entendimento.

A teoria da Física de partículas é algo não-trivial e que, de certa forma, é alheia aos estudantes, mesmo a nível de divulgação científica, devido a falta de uma política educacional de inserção no meio científico. Além disso, existe uma necessidade de aprofundamento teórico por parte dos professores em temas relacionados à Física Moderna, como destaca Ostermann e Ricci [62]. Novas condições metodológicas relacionadas à Física de Partículas precisam ser implementadas tanto a nível de graduação como a nível básico de ensino para que essa temática possa estar presente na prática diária docente. Podemos ver que na Europa, onde está localizado o LHC, vários pesquisadores estão elaborando propostas para ensinar Física de altas energias e inserir as partículas elementares no universo dos estudantes 63 65.

Criar condições metodológicas para os docentes perpassa pela melhoria da infraestrutura das escolas públicas, o que inclui reforma nas salas de aula, construção de laboratórios didáticos equipados, incentivo à realização de atividades lúdicas, formação continuada dos professores, valorização da carreira de magistério e eventos periódicos que tratem da Física de Altas Energias a nível de divulgação científica. Há espaços como a Semana Nacional de Ciência e Tecnologia(SNCT) onde se poderia tratar desta temática, que ocupa a vanguarda das pesquisas científicas e tem sim utilidade social e cultural, pois a ciência faz parte da cultura de um país desenvolvido e acreditamos que este é o caminho a ser trilhado, embora ele seja árduo e tortuoso.

Apesar destas barreiras, o jogo foi bem sucedido, devido a estratégia adotada. O objetivo do jogo não é avaliar o conhecimento dos estudantes e sim proporcionar aprendizado, de modo que no baralho de cartas havia perguntas de diversos níveis, e sempre que um jogador errava uma pergunta, os monitores do jogo explicavam a pergunta e discutiam a resposta. A carta era colocada novamente no baralho de modo que em outra rodada, caso a pergunta ocorresse novamente, o estudante estaria apto a respondê-la, e dentro desse aspecto funcionaria também como instrumento de avaliação. Utilizando a linha de pensamento construtivista, o erro é visto não como fracasso, mas como trampolim para o aprendizado.

Em uma experiência realizada entre estudantes do curso de Licenciatura em Física do IFPI, na disciplina de Núcleos Temáticos, as partidas mostraram-se mais dinâmicas e os alunos não tiveram dificuldades em jogar, enfatizando é claro que uma abordagem diferente foi realizada, uma vez que nesta disciplina eram explorados diversos tópicos de Pesquisa em Física, como Cosmologia, Astrofísica, Astronomia, Nanotecnologia, Física Nuclear, Física Médica, Matéria Condensada e Física de Partículas, ou seja, os estudantes já haviam estudado o tema e o jogo foi utilizado como complementação de aprendizagem e instrumento de avaliação. O jogo teve grande aceitação por parte dos estudantes do curso de Licenciatura em Física, onde estes relataram satisfação em conseguir concluir o jogo sem dificuldades e contextualizar os conhecimentos adquiridos com o ambiente do tabuleiro, que tem como pano de fundo o LHC, onde foi descoberto o bóson de Higgs.

Destacamos ainda que o jogo também pode ser disputado em grupos, estimulando a cooperação e o trabalho em equipe, possibilitando também troca de informações e debates entre os participantes. Aconselhamos a leitura de mais fontes sobre o tema, uma vez que neste artigo apresentamos alguns conceitos e definições de forma breve. Como exemplo da experiência feita no Ensino Superior, o jogo tornar-se-á mais efetivo no Ensino Médio ou na formação de docentes se for feito um estudo prévio dos fenômenos e teorias envolvidas, mesmo a nível de divulgação científica. Na literatura moderna existem vários artigos e textos interessantes abordando este tema e com enfoque voltado ao Ensino de Física e Ciência em geral 66 70 e que podem servir em conjunto com este trabalho como alternativa e proposta metodológica lúdica para o Ensino de Física de partículas.

\section{Considerações finais}

As vertentes mais modernas sobre educação defendem um ensino voltado para a construção de uma mentalidade dinâmica e criativa no aluno e com base nesse aspecto, este trabalho explorou a possibilidade da utilização de um jogo didático sobre Física de partículas ser trabalhado no Ensino Médio e também no Ensino Superior, visando a formação de professores. A temática é abordada sem perda de generalidade prática, uma vez que a estrutura conceitual e filosófica dos fenômenos, sob certo aspecto, pode ser mantida sem recorrer a fórmulas matemáticas e sempre, na medida do possível, expondo aplicações práticas que podem ser identificadas pelo aluno no cenário científico atual. Nesse ponto, faz-se necessária a intervenção do educador como uma ponte entre o saber teórico e conhecimento prático e contextual. Fica como sugestão para os educadores a utilização de recursos didáticos como animações e vídeos, hoje em dia tão difundidos na internet, que tratem desses assuntos. De modo geral, a ciência deve ser divulgada entre os jovens com o objetivo de despertá-los para a realidade da sociedade tecnológica, servindo como um veículo de inclusão e como forma de estímulo para formação de novos cientistas. 
Os autores agradecem à CAPES pelo apoio financeiro. $\mathrm{E}$ aos referees pelas sugestões de melhoria, que contribuiram para o enriquecimento deste trabalho.

\section{Material suplementar}

O seguinte material suplementar está disponível online: Apêndice A: Glossário de termos técnicos.

\section{Referências}

[1] A.S.T. Pires, Evolução das ideias da Física (Livraria da Física, São Paulo, 2011.), $2^{\mathrm{a}}$ ed.

[2] T. Mayer-Kuckuk, Física Nuclear, (Fundação Calouste Gulbenkian, Lisboa, 1993).

[3] E. Segré, Núcleos y Partículas (Editorial Reverte, Barcelona, 1972).

[4] K.S. Krane, Introductory Nuclear Physics (John Wiley \& Sons, New York, 1988)

[5] W.N. Cottingham e A.D. Greenwood, An Introduction to the Standard Model of Particle Physics (Cambridge university press, Cambridge, 2007).

[6] J. Goldstone, A. Salam e S. Weinberg, Phys. Rev. 127, 965 (1962).

[7] P. Higgs, Phys. Rev. Lett. 13, 508 (1964).

[8] W. Heisenberg, Zeitschrift für Physik 33, 879 (1925).

[9] E. Schrödinger, Phys. Rev. 28, 1049 (1926).

[10] P. Dirac, Proceedings of the Royal Society A 117, 610 (1928).

[11] D. Gross e F. Wilczek, Phys. Rev. Lett. 30, 1343 (1973).

[12] H. Politzer, Phys. Rev. Lett. 30, 1346 (1973).

[13] R. Feynman, Phys. Rev. 74, 1430 (1948).

[14] R. Feynman, Phys. Rev. 76, 769 (1949).

[15] J. Schwinger, Phys. Rev. 74, 1439 (1948).

[16] S. Tomonaga, Progr. Theor. Phys. 1, 27 (1946).

[17] S. Weinberg, Phys. Rev. Lett. 19, 1264 (1967).

[18] A. Salam, Proceedings of the Eighth Nobel Symposium, 367 Estocolmo, 1968.

[19] S. Glashow, Nuclear Physics 10, 107 (1959).

[20] W. Zeichner e J.E.D. Pereira, A pesquisa na formação e no trabalho docente (Autêntica, Belo Horizonte, 2002).

[21] E.C. Ricardo, em: Ensino de Física organizado por A.M.P. Carvalho (Cengage Learning, São Paulo, 2010), p. 29.

[22] S. Camargo e R. Nardi, em IX Encontro Nacional de Pesquisa em Ensino de Física, Jaboticatubas, 2004 (Sociedade Brasileira de Física, São Paulo, 2004), p. 1.

[23] C.M.M. Almeida, T.R. Prochnow e P.T.C. Lopes, Revista Góndola, Enseñanza y Aprendizaje de las Ciencias 11, 228 (2016).

[24] E. Fermi, Zeitschrift für Physik 88, 161 (1934).

[25] R.P. Feynman e M. Gell-Mann, Phys. Rev. 109, 193 (1958).

[26] M. Sanchez, S. Landsberger e J. Braisted, Journal of Radioanalytical and Nuclear Chemistry 269, 487 (2006).

[27] T.E. Driscol e R.M. Bern, Experimental Biology and Medicine 96, 505 (1957).

[28] http://www.searadaciencia.ufc.br/folclore/folclore131.htm, acessado em 20/06/2018.

[29] W. Heisenberg, Zeitschrift für Physik 77, 1 (1932).

[30] H. Yukawa, Proc. Phys.-Math. Soc. Japan 17, 48 (1935).
[31] M. Gell-Mann, Phys. Rev. 125, 1067 (1962).

[32] M. Gell-Mann, Physics Letters 8, 214 (1964).

[33] P.R. Silva, Rev. Bras. Ensino Fís. 30, 3305 (2008).

[34] M.A. Moreira, Textos de apoio ao professor de física (Universidade Federal do Rio Grande do Sul, Porto Alegre, 2017).

[35] Y. Nambu, Phys. Rev. Lett. 4, 380 (1960).

[36] Y. Nambu e G. Jona-Lasinio, Phys. Rev. 122, 345 (1961).

[37] E. Noether, Math-phys, 235 (1918).

[38] W. Su, J. Schrieffer e A. Heeger, Phys. Rev. Lett. 42, 1698 (1979).

[39] H. Nielsen e P. Olsen, Nucl. Phys. B 61, 45 (1973).

[40] G.'t Hooft, Nuclear Physics B 79, 276 (1974).

[41] A.M. Polyakov, Zh. Eksp. Teor. Fiz. Pis'ma. Red. 20, 430 (1974).

[42] T.W.B. Kibble, J. Phys. A 9, 1387 (1976).

[43] N. Turok, Phys. Rev. Lett. 63, 2625 (1989).

[44] J. Goldstone, Nuovo Cimento 19, 154 (1961).

[45] Y. Nambu e G. Jona-Lasinio, Phys. Rev. 124, 246 (1961).

[46] https://www.dropbox.com/s/b1nvkh9ecpie8c1/Jogo $\% 20$

Descobrindo\%20o\%20b\%C3\%B3son\%20de\%20Higgs.rar? $\mathrm{dl}=0$

[47] https://en.wikipedia.org/wiki/Large_Hadron_Collider, acessado em 20/03/2018.

[48] https://en.wikipedia.org/wiki/Color_charge, acessado em 20/03/2018.

[49] https://en.wikipedia.org/wiki/W_and_Z_bosons, acessado em 20/03/2018.

[50] P. Higgs, Physics Letters 12, 132 (1964).

[51] P. Higgs, Phys. Rev. 145, 1156 (1966).

[52] W.B. Fraga, J.R.R. Duarte, M.A.M Souza e A.R. Silva, Latin - American Journal of Physics Education 6, 418 (2012).

[53] M.A.M Souza, J.J. Rodrigues e A.R. Silva, Latin American Journal of Physics education 7, 357 (2013).

[54] M.A.M Souza e J.R.R. Duarte, Physics Education 50, $482(2015)$.

[55] E.A. Terrazzan, Caderno Catarinense de Ensino de Física 9, 209 (1992).

[56] G.J. Aubrecht, American Journal of Physics 57, 352 (1989).

[57] F. Ostermann e M.A. Moreira, A review on the "Modern and Contemporary Physics at High School" research area, disponível em: http://www.if.ufrgs.br/public/ensino/vol5/n1/v5_n1_a2. htm, acessado em 15/04/2018.

[58] http://phet.colorado.edu/index.php, acessado em $15 / 04 / 2018$

[59] M. Webb e D. Hassen, em: Computers in education editado por F. Lovis e E.D. Tagg (North-Holland, Amsterdam, 1988).

[60] M.A. Moreira, A teoria da Aprendizagem Significativa e sua implementação em sala de aula (Editora da UnB, Brasília, 2006).

[61] A. Paivio, Mind and Its Evolution: A Dual Coding Theoretical Approach (Lawrence Erlbaum, New Jersey, 2006).

[62] F. Ostermann e T.F. Ricci, Cad. Bras. Ens. Fís. 22, 9 (2005).

[63] J. Woithe, G.J. Wiener, J. Gerfried e F.F. Van der Veken, Phys. Educ. 52, 034001 (2017). 
[64] G.J. Wiener, J. Woithe, A. Brown e K. Jende, Phys. Educ. 51, 035001 (2016).

[65] G.J. Wiener, S.M. Schmeling e M. Hopf, Phys. Educ. 52, 044001 (2017).

[66] L. Evans, New J. Phys. 9, 335 (2007).

[67] F.K. Nóbrega e L.F. Mackedanz, Rev. Bras. de Ens. de Fis. 35, 1301 (2013).

[68] M.M. Pereira, Física na escola 12, 37 (2011).

[69] M.A. Moreira, Rev. Bras. de Ens. de Fis. 31, 1306 (2009).

[70] http://www.searadaciencia.ufc.br/folclore/folclore00.htm, acessado em 20/06/2018.

[71] C.R.S.L. Chakur, A desconstrução do Construtivismo na educação: crenças e equívocos de professores, autores e críticos, (Editora UNESP, São Paulo, 2014), p. 16.

[72] L. Landau, Phys. Rev. 60, 356 (1941).

[73] L. Landau, Phys. Rev. 75, 884 (1949).

[74] W. Zurek, Phys. Rep. 276, 177 (1996).

[75] S. Weinberg, Cosmology (Oxford University Press, Oxford, 2008).

[76] R.A. Alpher, H. Beth e G. Gamow, Phys. Rev. 73, 803 (1948).

[77] G. Gamow, Physical Review 74, 505 (1948).

[78] G. Gamow, Nature 162, 680 (1948).

[79] A.A. Penzias e R.W. Wilson, Astrophysical Journal 142, 419 (1965).

[80] S. Feeney, M.C. Johnson, D.J. Mortlock e H.V. Peiris, Phys. Rev. Lett. 108, 241301 (2012). 